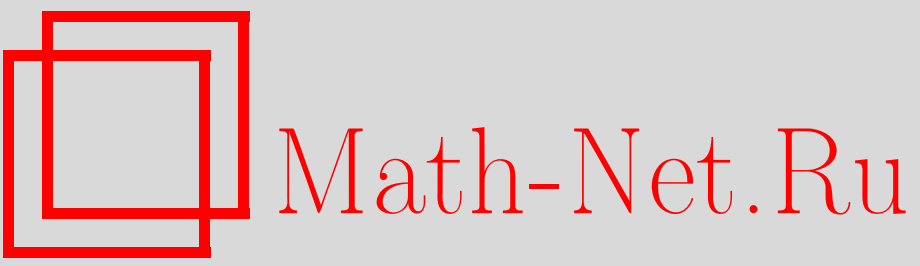

М. Ауади, К. Булеми, Граничная управляемость для неоднородной многомерной задачи термоупругой диффузии методом единственности Гильберта, Итоги науки и техн. Сер. Соврем. мат. и ее прил. Темат. обз., 2020, том 178, 57-76

DOI: https://doi.org/10.36535/0233-6723-2020-178-57-76

Использование Общероссийского математического портала Math-Net.Ru подразумевает, что вы прочитали и согласны с пользовательским соглашением

http://www.mathnet.ru/rus/agreement

Параметры загрузки:

IP: 54.89 .56 .158

26 апреля 2023 г., 10:35:29 


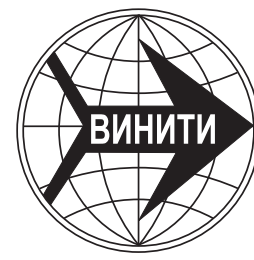

ИТОГИ НАУКИ И ТЕХНИКИ.

Современная математика и ее приложения.

Тематические обзоры.

Том 178 (2020). C. $57-76$

DOI: $10.36535 / 0233-6723-2020-178-57-76$

УДК 517.977.56

\title{
ГРАНИЧНАЯ УПРАВЛЯЕМОСТЬ ДЛЯ НЕОДНОРОДНОЙ МНОГОМЕРНОЙ ЗАДАЧИ ТЕРМОУПРУГОЙ ДИФФУЗИИ МЕТОДОМ ЕДИНСТВЕННОСТИ ГИЛЬБЕРТА
}

\author{
(c) 2020 г. \\ М. АУАДИ, К. БУЛЕМИ
}

\begin{abstract}
АннотАция. В статье исследуется вопрос стабилизации и частичной точной управляемости для неоднородной многомерной задачи термоупругой диффузии при помощи функции памяти, заданной на части границы материала. На основе разностного неравенства Накао доказано, что энергия системы экспоненциально уменьшается до нуля со скоростью, явно определяемой физическими параметрами. Затем при помощи метода единственности Гильберта в сочетании с принципом Расселла «управляемость через стабилизируемость» мы доказываем, что после определенного порогового момента времени рассматриваемая система частично управляема при ограничении на параметры напряжения-температуры и напряжения-диффузии. Граничная функция управления найдена в явном виде.
\end{abstract}

Ключевые слова: термоупругая диффузия, экспоненциальное убывание, граничная память, управляемость.

\section{BOUNDARY CONTROLLABILITY FOR INHOMOGENEOUS MULTIDIMENSIONAL THERMOELASTIC DIFFUSION PROBLEM BY HILBERT'S UNIQUENESS METHOD}

\author{
(c) 2020 M. AOUADI, K. BOULEHMI
}

\begin{abstract}
In this paper, we study the stabilization and the partial exact controllability of an inhomogeneous multidimensional thermoelastic diffusion problem by a memory function introduced on a part of the boundary of the material. By using the Nakao difference inequality, we prove that the energy of the system decays to zero exponentially at a rate determined explicitly by the physical parameters. Then by the Hilbert uniqueness method combined with Russell's principle "controllability via stabilizability" we prove that after certain threshold time moment the considered system is partially controllable under a smallness restriction on the coupling parameters stress-temperature and stressdiffusion. The boundary control function is determined explicitly.
\end{abstract}

Keywords and phrases: thermoelastic diffusion, exponential decay, boundary memory, controllability. AMS Subject Classification: 34H15, 93B05

1. Введение. Термоупругие материалы используются в некоторых областях техники, например, в металлургической промышленности, где эти материалы обрабатываются при сильных температурных градиентах. Важным примером является термомеханическое моделирование алюминиевого электролизера (см. [8]). Другим важным применением этого вида материалов является термомеханическое моделирование слябов при прямом охлаждении отливок из сплавов (см. [7]). В таких процессах очень важно учитывать механическое и тепловое рассеяние и неоднородность 
материалов. Из-за этих аспектов анализ стабилизации и управляемости таких процессов становится весьма трудным. По этой причине определение качественных свойств решений для термоупругих систем становится сложной математической задачей, в частности, когда коэффициенты материала зависят от пространственной координаты.

Исследования, проведенные в области развития высоких технологий после Второй мировой войны, подтвердили, что процесс диффузии в твердых телах нельзя игнорировать. Диффузия может быть определена как случайное блуждание множества частиц из областей с высокой концентрацией в области с более низкой концентрацией. Термодиффузия в упругом твердом теле обусловлена сочетанием полей деформации, температуры и диффузии массы. Процессы диффузии тепла и массы играют важную роль во многих инженерных приложениях. В этой статье мы изучаем влияние масс-диффузионной проводимости на стабилизацию и управляемость для неоднородного и анизотропного термоупругого материала.

В [9] было доказано, что энергия неоднородной термоупругой диффузионной системы, связанной с различными граничными условиями, экспоненциально убывает до нуля для одномерной задачи. Для многомерной задачи ситуация иная. В действительности степень свободы векторного поля смещений превосходит степень свободы температуры и диффузии, когда размерность больше чем $n=1$. В [3] исследована задача стабилизации термоупругой диффузии в неоднородной многомерной среде с помощью нелинейной функции обратной связи.

В теории термоупругости, начиная с новаторской работы [10], хорошо известно, что градиент температуры всегда убывает до нуля и что, как правило, смещение также убывает до нуля; однако скорость убывания оставалась неизвестной. В [16] приведено достаточное и необходимое условие, гарантирующее, что энергия стремится к нулю экспоненциально при $t \rightarrow \infty$ в ограниченной многомерной гладкой области $\Omega$. Из результата [16] следует, что если $\Omega$ ограничена гладким выпуклым открытым множеством, энергия не убывает до нуля экспоненциально. Таким образом, невозможно получить равномерную скорость убывания в общей ситуации. Это означает, что в нашем случае диссипация, обусловленная тепловым и диффузионным эффектами, не является достаточно сильной, чтобы обеспечить равномерное затухание термоупругой диффузионной системы более высокой размерности (см. (2.6)). Таким образом, для обеспечения равномерного затухания системы необходим дополнительный демпфирующий механизм. Руководствуясь этими соображениями, многие граничные функции типа памяти вводятся для обеспечения равномерного затухания в однородных и неоднородных термоупругих системах (см., например, [4, 5, 12, 24]).

Как уже было сказано, основной задачей в этой статье является стабилизация неоднородной многомерной термоупругой диффузионной системы (см. (2.6)) с помощью граничной функции типа памяти на части границы материала,

$$
u_{i}=-g * C_{i j k l} u_{k, l} \nu_{j}
$$

где $u_{i}$ - вектор смещения, $g: \mathbb{R}^{+} \rightarrow \mathbb{R}$ - ядро памяти, $C_{i j k l}$ - тензорные поля, представляющие упругость материала, и $\nu$ - вектор внешней нормали к границе.

Граничное условие (1.1) позволяет обеспечить экспоненциальное убывание энергии для различных однородных термоупругих систем (см. [4, 12]), если ядро памяти затухает экспоненциально. В этой статье на основе метода множителей Лагранжа и разностного неравенства Накао (см. [25]) будет построена функция Ляпунова, эквивалентная энергии, и с ее помощью доказано экспоненциального убывание со скоростью, явно определяемой физическими параметрами.

Стабилизация линейных термоупругих систем исследовалась активно, но об управляемости известно относительно мало. В [27] доказана частичная точная граничная управляемость для термоупругой системы в $\mathbb{R}^{n}$. Позже этот результат был улучшен при помощи метода единственности Гильберта (см. [18]). Оба результата касаются управления только вектором смещения, независимо от значений температуры, и относятся к исследованию свойства так называемой частичной управляемости. Метод единственности Гильберта широко и эффективно применяется для исследования вопроса точной управляемости. Обоснованием метода единственности Гильберта является теорема Лакса-Мильграма об изоморфизме (см. [18]). В [13] доказано, что точная управляемость как перемещением, так и температурой возможна для одномерной термоупругой системы 
через управление только тепловой или механической компонентой на границе. По-видимому, метод Хансена неприменим в случае многомерного пространства. Таким образом, задача точной управляемости как перемещением, так и температурой в этом случае намного сложнее. В [34] эта задача решена при помощи введенного понятия точной приближенной управляемости, а именно, доказано, что если $T$ достаточно велико, то термоупругая система является точно-приближенно управляемой с функцией управления с носителем в окрестности границы $\Omega$; другими словами, смещение является точно управляемым, а температура - приближенно управляемой. Этот метод опирается помимо прочего на метод множителей Лагранжа, свойство компактности и теорему единственности Хольмгрена. В [11] получен аналогичный результат для случая термоупругих пластин. В [17] доказано, что линейная термоупругая система с периодическими граничными условиями является нуль-управляемой. В [3,20,21] при помощьи принципа Расселла (см. [31]) доказана частичную точную управляемость различных термоупругих систем с различными функциями управления. В [15] представлены различные методы управления некоторыми системами.

Основные новые результаты данной статьи состоят в следующем. Вопрос стабилизации и управляемости при слабых силах обратной связи вида (1.1) является интересной открытой проблемой. Насколько известно авторам, этот вопрос не исследовался для неоднородных анизотропных термоупругих систем с диффузией. Рассмотренная в настоящей работе система уравнений состоит из гиперболического уравнения, связанного с двумя параболическими уравнениями. Ввиду нелинейности граничных условий, это создает новые математические трудности в исследовании вопросов стабилизации и управляемости. Несмотря на то, что использованный подход основан на классических методах, потребовались некоторые модификации, чтобы учесть сложную связь уравнений, а также неоднородность и анизотропную природу материала. Фактически, рассматриваемая система не вкладывается непосредственно в рамки классических теорий.

Ключевым компонентом доказательства как экспоненциального убывания энергии, так и управляемости является неравенство, которому удовлетворяет резольвента некоторого заданного уравнения Вольтерра. Оно позволяет получить явные выражения для нелинейности, входящей в дифференциальное неравенство, определяющее убывание энергии и функцию управления. Явные выражения для скорости убывания и функции управления играют важную роль в выборе материала, приводящему к более быстрому убыванию с оптимальным временем управления. Это отражает актуальность исследования с физической точки зрения.

Статья организована следующим образом. В разделе 2 приведены основные уравнения, даны начальные предположения и описаны главные результаты статьи. Корректность постановки кратко описывается в разделе 3. В разделе 4 показано, что решение рассматриваемой задачи убывает до нуля с экспоненциальной скоростью. В завершение в разделе 5 установлено свойство частичной точной управляемости.

2. Основные уравнения и главные результаты. Рассмотрим задачу многомерной термоупругой диффузии для неоднородного анизотропного материала. Обозначим через $\mathrm{H}=\left[\mathrm{H}_{i_{1} \cdots i_{k}}\right]$ тензорное поле размерности $n^{k}, k=0,1,2, \ldots$, определенное над $\mathbb{R}^{n} \times(0, \infty), n \geqslant 1$. Будем обозначать частные производные по пространственной переменной через

а производные по времени - через

$$
\mathrm{H}_{i_{1} \cdots i_{k} j_{1} \cdots j_{s}}=\frac{\partial^{s} \mathrm{H}_{i_{1} \cdots i_{k}}}{\partial x_{j_{1}} \cdots \partial x_{j_{s}}}
$$

$$
\dot{\mathrm{H}}_{i_{1} \cdots i_{k}}=\frac{\partial \mathrm{H}_{i_{1} \cdots i_{k}}}{\partial t}, \quad \ddot{\mathrm{H}}_{i_{1} \cdots i_{k}}=\frac{\partial^{2} \mathrm{H}_{i_{1} \cdots i_{k}}}{\partial t^{2}}, \quad \mathrm{H}_{i_{1} \cdots i_{k}}^{(s)}=\frac{\partial^{s} \mathrm{H}_{i_{1} \cdots i_{k}}}{\partial t^{s}}, \quad s \geqslant 3 .
$$

Примем соглашение о суммировании по повторяющемуся индексу, т.е.

$$
\mathrm{H}_{i_{k}} \mathrm{G}_{i_{k}}=\sum_{i_{k}} \mathrm{H}_{i_{k}} \mathrm{G}_{i_{k}}
$$

Обозначим через $\Omega \subset \mathbb{R}^{n}$ открытую ограниченную область, задающую конфигурационное пространство рассматриваемого материала, с границей $\Gamma=\partial \Omega$ класса $\mathcal{C}^{2}$. Для каждой точки $x$ в момент времени $t$ обозначим соответственно через $u, \theta$ и $P$ смещение, термический и химический 
потенциал. Обозначим через $\nu(x), x \in \Gamma=\partial \Omega$ вектор внешней нормали к границе Г. Будем предполагать, что существуют такие точка $x_{0} \in \mathbb{R}^{n}$ и векторное поле $q(x)=x-x_{0}$, что

$$
\Gamma_{0}=\{x \in \Gamma, q(x) \cdot \nu(x)>0\}, \quad \Gamma_{1}=\{x \in \Gamma, q(x) \cdot \nu(x)<0\} .
$$

Здесь $q(x) \cdot \nu(x)$ - скалярное произведение векторов $q(x)$ и $\nu(x)$ в пространстве $\mathbb{R}^{n}$. Заметим, что оба множества $\Gamma_{0}$ и $\Gamma_{1}$ являются непустыми и удовлетворяют соотношениям

$$
\overline{\Gamma_{0}} \cap \overline{\Gamma_{1}}=\emptyset, \quad \Gamma=\Gamma_{0} \cup \Gamma_{1} .
$$

Поскольку $\Gamma_{0}$ является компактным подмножеством, то существуют две положительные константы $a_{0}, a_{1}$, для которых выполнено условие

$$
0<a_{0} \leqslant q(x) \cdot \nu(x) \leqslant a_{1} \quad \text { для всех } x \in \Gamma_{0} .
$$

Обозначим через

$$
R_{0}=\max _{\bar{\Omega}}|q(x)|
$$

радиус наименьшего шара с центром в точке $x_{0}$, содержащего $\Omega$. Пусть $\sigma$-такая наименьшая положительная константа, что

$$
\|\operatorname{div} u\|_{-1} \leqslant \sigma\|u\|_{L^{2}(\Omega)} \quad \text { для всех } u \in L^{2}(\Omega)
$$

(см. [20,21]). Уравнения термоупругой диффузии для неоднородного анизотропного материала в отсутствие внешних сил, тепловых и химических источников имеют следующий вид (см. [2])

$$
\begin{array}{ll}
\rho \ddot{u}_{i}=\left(C_{i j k l} u_{k, l}+\alpha_{i j} \theta+\beta_{i j} P\right)_{, j} & \text { в } \Omega \times(0, \infty), \\
c \dot{\theta}+d \dot{P}=\alpha_{i j} \dot{u}_{i, j}+\left(k_{i j} \theta_{, j}\right)_{, i} & \text { в } \Omega \times(0, \infty), \\
d \dot{\theta}+m \dot{P}=\beta_{i j} \dot{u}_{i, j}+\left(h_{i j} P_{, j}\right)_{, i} & \text { в } \Omega \times(0, \infty) .
\end{array}
$$

Рассмотрим следующие граничные условия:

$$
\begin{array}{ll}
u_{i}=0, & (x, t) \in \Gamma_{1} \times(0, \infty), \\
u_{i}=\phi_{i}, & (x, t) \in \Gamma_{0} \times(0, \infty), \\
\theta=P=0, & (x, t) \in \Gamma \times(0, \infty) .
\end{array}
$$

В первой части этой статьи функция $\phi$ определяется как

$$
\phi_{i}=-g * C_{i j k l} u_{k, l} \nu_{j},
$$

где $g: \mathbb{R}^{+} \rightarrow \mathbb{R}$ - ядро памяти. Во второй части функция $\phi_{i}$ определяется так, чтобы получить частичную точную управляемость системы. В работе предполагается, что начальные условия имеют вид

$$
u(x, 0)=u^{0}(x), \quad \dot{u}(x, 0)=u^{1}(x), \quad \theta(x, 0)=\theta^{0}(x), \quad P(x, 0)=P^{0}(x), \quad x \in \Omega .
$$

В приведенных выше уравнениях $\rho(x)$ обозначает плотность массы в точке $x \in \Omega$, а $C_{i j k l}(x)$, $\alpha_{i j}(x), \beta_{i j}(x)$ - тензорные поля, представляющие упругость материала, тепловое напряжение и диффузионное напряжение. Через $c(x), d(x)$ и $m(x)$ обозначены соответственно удельная теплоемкость, мера термодиффузионного эффекта и мера диффузионного эффекта. Через $k_{i j}(x)$ обозначен тензор теплопроводности, а через $h_{i j}(x)$ - тензор проводимости диффузионного поля.

Предполагается, что $C_{i j k l}(x), \alpha_{i j}(x), \beta_{i j}(x), k_{i j}(x)$ и $h_{i j}(x)$ являются гладкими на $\bar{\Omega}$ и удовлетворяют следующим условиям:

$$
\begin{gathered}
C_{i j k l}=C_{j i k l}=C_{k l i j}, \\
\alpha_{i j}=\alpha_{j i}, \quad \beta_{i j}=\beta_{j i}, \quad k_{i j}=K_{j i}, \quad h_{i j}=h_{j i} .
\end{gathered}
$$

Существуют такие положительные константы $\gamma_{1}, \alpha, \varsigma$ и $\kappa$, что

$$
\begin{aligned}
& \left\{C_{i j k l}-q_{\mu} C_{i j k l, \mu}\right\} \xi_{i j} \xi_{k l} \geqslant \gamma_{1} \xi_{i j} \xi_{i j}, \\
& C_{i j k l} u_{k, l} u_{i, j} \geqslant \alpha u_{i, j}^{2},
\end{aligned}
$$


а также

$$
\int_{\Omega} \theta_{, i} k_{i j} \theta_{, j} d x \geqslant \varsigma \int_{\Omega} \theta_{, i}^{2} d x, \quad \int_{\Omega} P_{, i} h_{i j} P_{, j} d x \geqslant \kappa \int_{\Omega} P_{, i}^{2} d x
$$

Определим следующие величины:

$$
\begin{gathered}
\omega_{1}:=\max _{\{x, i, j\}}\left|\alpha_{i j}(x)\right|, \quad \omega_{2}:=\max _{\{x, i, j\}}\left|\beta_{i j}(x)\right|, \quad \omega_{3}:=\max _{\{x, i, j\}}\left|\alpha_{i j, j}(x)\right|, \\
\omega_{4}:=\max _{\{x, i, j\}}\left|\beta_{i j, j}(x)\right|, \quad \omega_{5}:=\max _{\{x, i, j\}}\left|C_{i j k l}(x)\right|, \quad x \in \bar{\Omega} .
\end{gathered}
$$

Плотность $\rho \in C(\bar{\Omega}) \cap W^{1, \infty}(\Omega)$ удовлетворяет следующим условиям:

$$
\begin{gathered}
\rho_{, j} \in L^{\infty}(\Omega), \\
0<\rho_{0}<\rho(x)<\rho_{1}, \\
\rho(x)+q_{\mu}(x) \rho_{, \mu}(x)>\gamma_{0},
\end{gathered}
$$

где $\gamma_{0}$ - положительная константа. Функции $c(x), m(x)$ и $d(x)$ непрерывны на $\bar{\Omega}$ и удовлетворяют условиям

$$
0<c_{0}<c(x)<c_{1}, \quad 0<d_{0}<d(x)<d_{1}, \quad 0<m_{0}<m(x)<m_{1} .
$$
Симметричная матрица $\mathcal{M}(x)=\left(\begin{array}{cc}c(x) & d(x) \\ d(x) & m(x)\end{array}\right)$ является равномерно положительно определен-
ной на $\Omega$, т.е.

$$
\Delta(x)=c(x) m(x)-d^{2}(x)>0 \quad \text { для всех } x \in \Omega .
$$

Отметим, что условие (2.15) влечет

$$
c(x) \theta^{2}(x)+2 d(x) \theta(x) P(x)+m(x) P^{2}(x)>0 \quad \text { для всех } x \in \Omega .
$$

Это условие необходимо для стабилизации термоупругой диффузионной системы (см. [2]).

Дифференцируя граничное условие $(2.7 \mathrm{~b})$ по $t$ и используя $(2.8)$, получим уравнение

$$
\dot{u}_{i}+g(0) C_{i j k l} u_{k, l} \nu_{j}+\int_{0}^{t} g^{\prime}(t-\tau) C_{i j k l} u_{k, l} \nu_{j} d \tau=0
$$

которое эквивалентно следующему:

$$
C_{i j k l} u_{k, l} \nu_{j}=-\frac{1}{g(0)} \dot{u}_{i}-\int_{0}^{t} \frac{1}{g(0)} g^{\prime}(t-\tau) C_{i j k l} u_{k, l} \nu_{j} d \tau
$$

Чтобы построить решение уравнения (2.16), используется резольвента $r(t)$ уравнения Вольтерра

$$
C_{i j k l} u_{k, l} \nu_{j}=-\frac{1}{g(0)} \dot{u}_{i}-r * \dot{u}_{i} .
$$

Резольвента $r(t)$ является решением интегрального уравнения

$$
g(0) r(t)+g^{\prime}(t)+\int_{0}^{t} g^{\prime}(t-\tau) r(\tau) d \tau=0 .
$$

Предполагается, что

$$
\begin{gathered}
g(t), g^{\prime}(t) \in L A C \text { (локально абсолютно непрерывны) }, \\
g(0)>0, g(t), \quad g^{\prime \prime}(t) \in L_{\mathrm{loc}}^{1}\left(\mathbb{R}^{+}\right) .
\end{gathered}
$$

Из теории уравнений Вольтерра известно, что

$$
r, r^{\prime} \in L_{\text {loc }}^{1}\left(\mathbb{R}^{+}\right) .
$$


Определение 2.1 (см. [28]). (i) Функция $\wp \in L_{\text {loc }}^{1}\left(\mathbb{R}^{+}, \mathbb{C}^{n \times n}\right)$ называется положительной, если для любой функции $\varphi \in L^{2}\left(\mathbb{R} ; \mathbb{C}^{n}\right)$ с компактным носителем выполнено

$$
\operatorname{Re}\left(\int_{\mathbb{R}}\langle\varphi(t),(\wp * \varphi)(t)\rangle d t\right) \geqslant 0 .
$$

(ii) Функция $\wp \in L_{\text {loc }}^{1}\left(\mathbb{R}^{+}, \mathbb{C}^{n \times n}\right)$ называется сильно положительной, если существует константа $c>0$, для которой функция $\ell(t)=\wp(t)-c e^{-t}$ является положительной.

Напомним некоторые утверждения, которые будут использованы в последующих разделах.

Лемма 2.1 (см. [33]). Пусть функиия $\wp \in L_{\mathrm{loc}}^{1}\left(\mathbb{R}^{+}\right)$является сильно положительной и $\wp^{\prime} \in$ $L_{\mathrm{loc}}^{1}\left(\mathbb{R}^{+}\right)$. Для любой функиии $y \in L_{\mathrm{loc}}^{1}\left(\mathbb{R}^{+}\right)$выполнено неравенство

$$
\int_{t_{0}}^{t_{1}}|\wp * y(\tau)|^{2} d \tau \leqslant \mathfrak{B}_{0} \mathfrak{B}_{\wp} \int_{t_{0}}^{t_{1}}(\wp * y(\tau)) y(\tau) d \tau, \quad 0 \leqslant t_{0}<t_{1}<\infty,
$$

гдe

$$
\mathfrak{B}_{\wp}=\left(\int_{0}^{+\infty}|\wp(t)| d t\right)^{2}+4\left(\int_{0}^{+\infty}\left|\wp^{\prime}(t)\right| d t\right)^{2},
$$

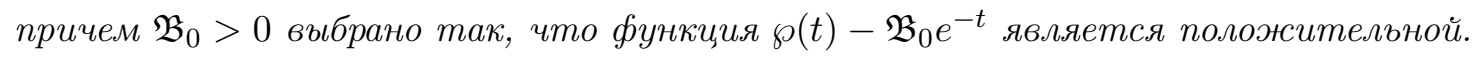

Замечание. 1. Неравенство (2.21) является модификацией леммы 1 и теоремы 2(ii) из [32].

2. Поскольку ядро $r(t)$ - решение уравнения $(2.18)$ - принадлежит классу $L_{\text {loc }}^{1}\left(\mathbb{R}^{+}\right)$и $r^{\prime}(t) \in$ $L_{\mathrm{loc}}^{1}\left(\mathbb{R}^{+}\right)$, далее в статье предполагается, что $r(t)$ удовлетворяет условию $(2.21)$. Это ключевой компонент в доказательстве экспоненциального убывания и управляемости в рассматриваемой задаче.

Для доказательства экспоненциальной устойчивости далее потребуется разностное уравнение Накао (см. [25]).

Лемма 2.2. Пусть функиия $\Phi(t):[0, \infty) \rightarrow[0, \infty)$ является локально абсолютно непрерывной и невозрастающей и пусть существуют такие две положительные константы $b$ и $ш$, что

$$
\sup _{t \leqslant s \leqslant t+1} \Phi^{b+1}(s) \leqslant \varpi(\Phi(t)-\Phi(t+1)), \quad t>0 .
$$

Eсли $b=0, m o$

$$
\Phi(t) \leqslant \Phi(0) e^{-t / \varpi}, \quad t>0 .
$$

Если $b>0$, то существует такая положительная константа $C$ (зависящая от $\Phi(0)$ и $)$, что

$$
\Phi(t) \leqslant C(1+t)^{-1 / b}, \quad t>0 .
$$

На основе приведенных результатов в разделе 4 будет доказано экспоненциальное убывание решения системы (2.6)-(2.9). Введем функцию энергии

$$
E(t)=E\left(u_{i}, \theta, P, t\right)=\frac{1}{2} \int_{\Omega}\left(\rho\left|\dot{u}_{i}\right|^{2}+C_{i j k l} u_{k, l} u_{i, j}+\mathcal{M}(x)\left(\begin{array}{c}
\theta \\
P
\end{array}\right) \cdot\left(\begin{array}{c}
\theta \\
P
\end{array}\right)\right) d x .
$$

Теорема 2.1. Пусть резольвента $r(t)$, заданная уравнением (2.18), удовлетворяет условию (2.21), и пусть выполнены предположения (2.10)-(2.15). Тогда существуют такие положительные константы $\Lambda>1 u \omega>0$, что

$$
E(t) \leqslant \Lambda E(0) e^{-\omega t},
$$


где

$$
\begin{gathered}
\Lambda=\frac{\delta+\varrho_{0}}{\delta-\varrho_{0}}, \quad \omega=\frac{\Lambda_{1}}{\Lambda_{1}+\delta+\varrho_{0}}, \quad \Lambda_{1}=\min \left\{\gamma_{0}, \frac{\gamma_{1}}{2}-\epsilon \lambda_{1}, \delta \varsigma-\tau, \delta \kappa-\tau\right\}, \\
\delta=\max \left\{\frac{(n-1)^{2}}{2 \epsilon g(0)}+g(0) \rho_{1} a_{1}+\frac{g(0) R_{0}^{2}}{2 \epsilon}, \frac{(n-1)^{2} \mathfrak{B}_{r} \mathfrak{B}_{0}}{2 \epsilon g^{2}(0)}+\frac{R_{0}^{2} \mathfrak{B}_{r} \mathfrak{B}_{0}}{2 \epsilon g^{2}(0)}, \frac{\tau}{\varsigma}, \frac{\tau}{\kappa}, \varrho_{0}+1\right\}, \\
\varrho_{0}=\max \left\{\frac{2 R_{0}}{\rho_{1}}+\frac{(n-1)}{\alpha}, \frac{R_{0} \rho_{1}^{2}}{2 \alpha}+(n-1) \frac{\rho_{1} C_{p}}{4}\right\}, \quad \epsilon=\min \left\{a_{0} \alpha, \frac{\gamma_{1}}{4 \lambda_{1}}\right\} .
\end{gathered}
$$

В разделе 5 с помощью метода единственности Гильберта (см. [18]) в сочетании с принципом Расселла «управляемость через стабилизируемость» (см. [31]) исследован вопрос управляемости рассматриваемой задачи, где $\phi=\left(\phi_{1}, \ldots, \phi_{n}\right)$ в $(2.7 \mathrm{~b})$ - неизвестная функция управления (которая будет определена позже), действующая на границе $\Gamma_{0}$. Сформулируем основной результат раздела 5, введя следующее функциональное пространство:

$$
H_{\Gamma_{1}}^{1}(\Omega)=\left\{\varphi \in H^{1}(\Omega) ; \varphi=0 \text { на } \Gamma_{1}\right\} .
$$

Теорема 2.2. Пусть резольвента $r(t)$ удовлетворяет уравнению (2.21) и

$$
\gamma \eta<\frac{\omega}{2 \sigma e \sqrt{\Lambda}}
$$

где

$$
\eta^{2}=2 \frac{\left(\omega_{3} C_{p}+\omega_{1}\right)^{2}}{\zeta}+2 \frac{\left(\omega_{4} C_{p}+\omega_{2}\right)^{2}}{\kappa}, \quad \gamma^{2}=\max \left\{\frac{\omega_{1}^{2}+\omega_{3}^{2} C_{p}}{\zeta}, \frac{\omega_{2}^{2}+\omega_{4}^{2} C_{p}}{\kappa}\right\},
$$

$\sigma, \Lambda$ и $\omega$ соответственно определены в (2.5) и (2.27). Пусть $T_{0}$ выбрано достаточно большим, так что выполнено неравенство

$$
2 \Lambda e^{\left(1-\omega T_{0}\right)}<1-\frac{4 \Lambda \eta^{2} \gamma^{2} \sigma^{2} e^{2}}{\omega^{2}}
$$

u $T \geqslant T_{0}$. Тогда для каљсдого состояния $\left(u^{0}, u^{1}\right) \in\left(H_{\Gamma_{1}}^{1}(\Omega)\right)^{n} \times\left(L^{2}(\Omega)\right)^{n}$ существует такая функиия граничного управления

$$
\phi_{i}(x, t) \in L^{2}\left(\Sigma_{0}\right), \quad \phi_{i}=-g * C_{i j k l}\left(w_{k, l}+z_{k, l}\right) \nu_{j},
$$

что решение задачи (2.6)-(2.7) с однородными начальными условиями удовлетворяет условиям

$$
u(x, T)=u^{0}(x), \quad \dot{u}(x, T)=u^{1}(x), \quad x \in \Omega .
$$

Более того, существует такая положительная постоянная $C$, не зависящая от $\left(u^{0}, u^{1}\right)$, что

$$
\|\phi\|_{\left(L^{2}\left(\Sigma_{0}\right)\right)^{n}} \leqslant C\left\|\left(u^{0}, u^{1}\right)\right\|_{\left(H_{\Gamma_{1}}^{1}(\Omega)\right)^{n} \times\left(L^{2}(\Omega)\right)^{n}} .
$$

3. Корректность постановки. В этом разделе на основе теории полугрупп будет кратко описана корректность постановки задачи (2.6)-(2.9).

В дальнейшем $H^{s}(\Omega), s \in \mathbb{R}$, обозначает обычное пространство Соболева (см. [1]). При $s \geqslant 0$ через $H_{0}^{s}(\Omega)$ обозначаем пополнение $C_{0}^{\infty}(\Omega)$ в $H^{s}(\Omega)$, где $C_{0}^{\infty}(\Omega)$ - пространство всех бесконечно дифференцируемых функций на $\Omega$ с компактным носителем в $\Omega$.

Пусть $X$ - банахово пространство. Обозначим через $C^{k}([0, T] ; X)$ пространство всех $k$ раз непрерывно дифференцируемых функций, определенных на $[0, T]$ со значениями в $X$. Для пространства $C^{0}([0, T] ; X)$ будем использовать обозначение $C([0, T] ; X)$.

При выполнении условий $(2.10 \mathrm{a}),(2.11 \mathrm{a})$ и $\Gamma_{1} \neq \emptyset$ легко показать, что норма

$$
\|\varphi\|_{\left(H_{\Gamma_{1}}^{1}(\Omega)\right)^{n}}=\frac{1}{2} \int_{\Omega} C_{i j k l} \varphi_{k, l} \varphi_{i, j} d x,
$$

пространства $\left(H_{\Gamma_{1}}^{1}(\Omega)\right)^{n}$ (см. (2.28)) эквивалентна стандартной норме, индуцированной $\left(H^{1}(\Omega)\right)^{n}$ (см. [20]). Поскольку $\Gamma_{1} \neq \emptyset$, определим функциональное пространство

$$
W=\left(H_{\Gamma_{1}}^{1}(\Omega)\right)^{n} \times\left(L^{2}(\Omega)\right)^{n} .
$$


Заметим, что норма

$$
\|(u, v)\|_{W}=\frac{1}{2} \int_{\Omega}\left(\rho|v|^{2}+C_{i j k l} u_{k, l} u_{i, j}\right) d x
$$

в $W$ эквивалентна стандартной норме, индуцированной $\left(H^{1}(\Omega)\right)^{n} \times\left(L^{2}(\Omega)\right)^{n}$.

Для любой функции $\varphi \in H_{\Gamma_{1}}^{1}(\Omega)$ выполнено неравенство Пуанкаре

$$
\|\varphi\|_{L^{2}(\Omega)} \leqslant C_{p}\left(\int_{\Omega} \varphi_{, j}^{2}\right)^{\frac{1}{2}}
$$

где $C_{p}>0$ - постоянная Пуанкаре. В силу неравенства для следа (см. [19]) существует такая положительная константа $\lambda_{1}$, что

$$
\int_{\Gamma_{0}}|\varphi|^{2} d \Gamma \leqslant \lambda_{1}\|\varphi\|_{\left(H_{\Gamma_{1}}^{1}(\Omega)\right)^{n}}^{2} \quad \text { для всех } \varphi \in\left(H_{\Gamma_{1}}^{1}(\Omega)\right)^{n} .
$$

Введем оператор $\mathcal{A}:=\left[\mathcal{A}_{i}\right]$ следующим образом:

$$
\mathcal{A}_{i}\left(\begin{array}{c}
u \\
v \\
\theta \\
P
\end{array}\right)=\left(\begin{array}{c}
v_{i} u_{i+} \frac{1}{\rho}\left(\alpha_{i j} \theta+\beta_{i j} P\right)_{, j} \\
m C_{i} \theta-d D_{i} P+\frac{1}{c m-d^{2}}\left(m \alpha_{i j}-d \beta_{i j}\right) v_{i, j} \\
-d C_{i} \theta+m D_{i} P+\frac{1}{c m-d^{2}}\left(c \beta_{i j}-d \alpha_{i j}\right) v_{i, j}
\end{array}\right),
$$

где

$$
A_{i} u_{i}=\frac{1}{\rho}\left(C_{i j k l} u_{k, l}\right)_{, j}, \quad C_{i} \theta=\frac{1}{c m-d^{2}}\left(k_{i j} \theta_{, j}\right)_{, i}, \quad D_{i} P=\frac{1}{c m-d^{2}}\left(h_{i j} P_{, j}\right)_{, i} .
$$

Теорема представлений Рисса гарантирует, что $A:=\left[A_{i}\right]$ является изометрическим изоморфизмом $\left(H_{\Gamma_{1}}^{1}(\Omega)\right)^{n}$ на $\left[\left(H_{\Gamma_{1}}^{1}(\Omega)\right)^{n}\right]^{\prime}, C:=\left[C_{i}\right]$ и $D:=\left[D_{i}\right]$ являются изометрическими изоморфизмами $H_{\Gamma_{1}}^{1}(\Omega)$ на $\left[H_{\Gamma_{1}}^{1}(\Omega)\right]^{\prime}$ (двойственное пространство к $H_{\Gamma_{1}}^{1}(\Omega)$ ).

Пусть $\langle\cdot, \cdot\rangle-$ скобки двойственности (спаривание) между $\left(H_{\Gamma_{1}}^{1}(\Omega)\right)^{n}$ и $\left[\left(H_{\Gamma_{1}}^{1}(\Omega)\right)^{n}\right]^{\prime}$ или $H_{0}^{1}(\Omega)$ и $H^{-1}(\Omega)$. Определим также нелинейный оператор $B$ формулой

$$
\langle B u, v\rangle=\int_{\Gamma_{0}}\left(-\frac{1}{g(0)} u-r * u\right) v d \Gamma \quad \text { для всех } u, v \in\left(H_{\Gamma_{1}}^{1}(\Omega)\right)^{n} .
$$

Заметим, что оператор $B:=\left[B_{i}\right]$ отображает $\left(H_{\Gamma_{1}}^{1}(\Omega)\right)^{n}$ на $\left[\left(H_{\Gamma_{1}}^{1}(\Omega)\right)^{n}\right]^{\prime}$ (см. [23]).

Используя введенные обозначения, заключаем, что задача (2.6)-(2.9) эквивалентна задаче Коши

$$
\left(\dot{u}_{i}, \dot{v}_{i}, \dot{\theta}, \dot{P}\right)=\mathcal{A}_{i}(u, v, \theta, P), \quad i=1, \ldots, n,
$$

или в векторной записи

$$
\begin{aligned}
& \frac{d U(t)}{d t}=\mathcal{A} U(t), \quad t>0, \\
& U(0)=\left(u^{0}, u^{1}, \theta^{0}, P^{0}\right),
\end{aligned}
$$

где нелинейный оператор $\mathcal{A}$ рассматривается в пространстве

$$
\mathcal{H}=W \times L^{2}(\Omega) \times L^{2}(\Omega),
$$

снабженном скалярным произведением

$$
(U, Y)_{\mathcal{H}}=\int_{\Omega}\left(C_{i j k l} u_{k, l} z_{i, j}+\rho v_{i} w_{i}+\mathcal{M}\left(\begin{array}{c}
\theta \\
P
\end{array}\right) \cdot\left(\begin{array}{l}
\phi \\
\psi
\end{array}\right)\right) d x,
$$

где $U=(u, v, \theta, P)$ и $Y=(z, w, \phi, \psi)$ из $\mathcal{H}$ таковы, что $v_{i}=\dot{u}_{i}$ и $w_{i}=\dot{z}_{i}$. 
Производная функции энергии (2.25) по времени может быть вычислена путем умножения (2.6a), $(2.6 \mathrm{~b})$ и $(2.6 \mathrm{c})$ соответственно на $v_{i}=\dot{u}_{i} \in H_{\Gamma_{1}}^{1}(\Omega), \theta, P \in H_{0}^{1}(\Omega)$ и интегрированием по области $\Omega$. Получаем

$$
\frac{d}{d t} E(t)=\int_{\Gamma_{0}} \nu_{j} C_{i j k l} u_{k, l} \dot{u}_{i} d \Gamma-\int_{\Omega} \theta_{, i} k_{i j} \theta_{, j} d x-\int_{\Omega} P_{, i} h_{i j} P_{, j} d x .
$$

Предполагаем, что выполнено условие (2.15). Используя (3.3) и (3.5), получаем

$$
\langle\mathcal{A} U, U\rangle_{\mathcal{H}}=\int_{\Gamma_{0}} \nu_{j} C_{i j k l} u_{k, l} \dot{u}_{i} d \Gamma-\int_{\Omega} \theta_{, i} k_{i j} \theta_{, j} d x-\int_{\Omega} P_{, i} h_{i j} P_{, j} d x=\frac{d}{d t} E(t) .
$$

Используя (2.17) и (2.12), находим

$$
\frac{d}{d t} E(t) \leqslant-\frac{1}{g(0)} \int_{\Gamma_{0}}\left|\dot{u}_{i}\right|^{2} d \Gamma-\int_{\Gamma_{0}} \dot{u}_{i}\left(r * \dot{u}_{i}\right) d \Gamma-\varsigma \int_{\Omega}\left|\theta_{, j}\right|^{2} d x-\kappa \int_{\Omega}\left|P_{, j}\right|^{2} d x,
$$

где функция $r(t)$ задана уравнением (2.18). Пусть $r(t)$ удовлетворяет (2.21); интегрируя (3.7) от 0 до $t$, получаем

$$
\begin{aligned}
E(t)-E(0) \leqslant-\frac{1}{g(0)} \int_{0}^{t} \int_{\Gamma_{0}}\left|\dot{u}_{i}\right|^{2} d \Gamma d \tau-\frac{1}{\mathfrak{B}_{0} \mathfrak{B}_{r}} & \int_{0}^{t} \int_{\Gamma_{0}}\left|r * \dot{u}_{i}\right|^{2} d \Gamma d \tau- \\
& -\varsigma \int_{0}^{t} \int_{\Omega}\left|\theta_{, j}\right|^{2} d x d \tau-\kappa \int_{0} \int_{\Omega}\left|P_{, j}\right|^{2} d x d \tau \leqslant 0 .
\end{aligned}
$$

Из (3.8) можно сделать вывод, что

$$
\langle\mathcal{A} U, U\rangle_{\mathcal{H}}=\frac{d}{d t} E(t) \leqslant 0,
$$

откуда следует диссипативность $\mathcal{A}$.

На основании указанных свойств операторов $A, B, C, D$ и соотношений $(2.1),(2.2),(2.18)$, $(2.20)$ и $(2.21)$, рассуждая аналогично $[3,9,23,24,29,30]$, стандартным способом можно показать, что $\mathcal{A}$ является $m$-диссипативным плотно определенным оператором и образует $C_{0}$-полугруппу сжатий $S(t)$ на $\mathcal{H}$. Это дает следующий результат о корректности постановки рассматриваемой задачи.

Теорема 3.1. Пусть $\Gamma_{0}$ и $\Gamma_{1}$ непусты и удовлетворяют условиям (2.1) и (2.2). Пусть функиии $g(t)$ и $r(t)$ удовлетворяют условиям (2.18), (2.20) и (2.21). Тогда для любого начального условия $\left(u^{0}, u^{1}, \theta^{0}, P^{0}\right) \in \mathcal{H}$ задача (2.6)-(2.9) имеет единственное решение

$$
\left(u, u_{t}, \theta, P\right)=S(t)\left(u^{0}, u^{1}, \theta^{0}, P^{0}\right) \in \mathcal{C}([0, \infty) ; \mathcal{H}) .
$$

Замечание. Эту теорему можно получить применением [30, теорема 4.4] в рамках предположений $(2.1),(2.2),(2.18),(2.20)$ и $(2.21)$.

4. Экспоненциальное убывание решения. Покажем, что решение задачи (2.6)-(2.9) убывает до нуля с экспоненциальной скоростью. Используемый для этого метод основан на построении функции $\mathcal{L}(t)$, эквивалентной функции энергии $(2.25)$, производная которой отрицательна. Перед доказательством теоремы 2.1 приведем несколько лемм.

Лемма 4.1. Для любого начального условия $\left(u^{0}, u^{1}, \theta^{0}, P^{0}\right) \in \mathcal{H}$, решение $(u, v, \theta, P)$ задачи (2.6)-(2.9) удовлетворяет уравнению

$$
\frac{d}{d t} \mathcal{F}(t)=\int_{\Gamma_{0}} \rho\left(q_{\mu} \cdot \nu_{\mu}\right)\left|\dot{u}_{i}\right|^{2} d \Gamma-\int_{\Omega}\left(n \rho+\rho_{, \mu} q_{\mu}\right)\left|\dot{u}_{i}\right|^{2} d x+2 \int_{\Gamma_{0}} q_{\mu} u_{i, \mu} C_{i j k l} u_{k, l} \nu_{j} d \Gamma+
$$




$$
\begin{array}{r}
+\int_{\Gamma_{1}}\left(q_{\mu} \cdot \nu_{\mu}\right) C_{i j k l} u_{k, l} u_{i, j} d \Gamma-\int_{\Gamma_{0}}\left(q_{\mu} \cdot \nu_{\mu}\right) C_{i j k l} u_{k, l} u_{i, j} d \Gamma+(n-2) \int_{\Omega} C_{i j k l} u_{k, l} u_{i, j} d x+ \\
+\int_{\Omega} q_{\mu} C_{i j k l, \mu} u_{k, l} u_{i, j} d x+2 \int_{\Omega}\left(\alpha_{i j} \theta\right)_{j} q_{\mu} u_{i, \mu} d x+2 \int_{\Omega}\left(\beta_{i j} P\right)_{, j} q_{\mu} u_{i, \mu} d x
\end{array}
$$

где

и $q_{\mu}-\mu-я$ компонента $q$.

$$
\mathcal{F}(t)=\int_{\Omega} 2 \rho q_{\mu} u_{i, \mu} \dot{u}_{i} d x, \quad \mu=1, \ldots, n
$$

Доказательство. Дифференцируя функцию $\mathcal{F}(t)$ по времени, получим

$$
\frac{d}{d t} \mathcal{F}(t)=2 \int_{\Omega} \rho q_{\mu} \dot{u}_{i, \mu} \dot{u}_{i} d x+2 \int_{\Omega} \rho q_{\mu} u_{i, \mu} \ddot{u}_{i} d x
$$

Интегрируя по частям, запишем первое слагаемое в правой части (4.2) в виде

$$
2 \int_{\Omega} \rho q_{\mu} \dot{u}_{i, \mu} \dot{u}_{i} d x=\int_{\Gamma_{0}} \rho q_{\mu} \cdot \nu_{\mu} \dot{u}_{i} \dot{u}_{i} d \Gamma-\int_{\Omega}\left(n \rho+\rho_{, \mu} q_{\mu}\right) \dot{u}_{i} \dot{u}_{i} d x ;
$$

второе слагаемое можно привести к виду

$$
\begin{aligned}
\int_{\Omega} \rho q_{\mu} u_{i, \mu} \ddot{u}_{i} d x=\int_{\Gamma} \nu_{j} C_{i j k l} u_{k, l} q_{\mu} u_{i, \mu} d \Gamma-\int_{\Omega} C_{i j k l} u_{k, l} u_{i, j} d x-\int_{\Omega} C_{i j k l} u_{k, l} q_{\mu} u_{i, j \mu} d x \\
\quad+\int_{\Omega}\left(\alpha_{i j} \theta\right)_{j} q_{\mu} u_{i, \mu} d x+\int_{\Omega}\left(\beta_{i j} P\right)_{, j} q_{\mu} u_{i, \mu} d x .
\end{aligned}
$$

Заметим, что симметрия по коэффициенту подразумевает выполнение равенства

$$
\left(C_{i j k l} u_{k, l} u_{i, j}\right)_{, \mu}=C_{i j k l, \mu} u_{k, l} u_{i, j}+2 C_{i j k l} u_{k, l} u_{i, j \mu} .
$$

Используя вышеупомянутое тождество, приведем третье слагаемое правой части равенства (4.4) к виду

$$
\begin{aligned}
\int_{\Omega} C_{i j k l} u_{k, l} q_{\mu} u_{i, j \mu} d x=\frac{1}{2} \int_{\Gamma}\left(q_{\mu} \cdot \nu_{\mu}\right) C_{i j k l} u_{k, l} u_{i, j} d \Gamma- & \\
& -\frac{n}{2} \int_{\Omega} C_{i j k l} u_{k, l} u_{i, j} d x-\frac{1}{2} \int_{\Omega} q_{\mu} C_{i j k l, \mu} u_{k, l} u_{i, j} d x .
\end{aligned}
$$

Подстановка (4.5) в (4.4) дает

$$
\begin{aligned}
& \int_{\Omega} \rho \ddot{u}_{i} q_{\mu} u_{i, \mu} d x=\int_{\Gamma} \nu_{j} C_{i j k l} u_{k, l} q_{\mu} u_{i, \mu} d \Gamma-\int_{\Omega} C_{i j k l} u_{k, l} u_{i, j} d x- \\
& -\frac{1}{2} \int_{\Gamma}\left(q_{\mu} \cdot \nu_{\mu}\right) C_{i j k l} u_{k, l} u_{i, j} d \Gamma+\frac{n}{2} \int_{\Omega} C_{i j k l} u_{k, l} u_{i, j} d x+\frac{1}{2} \int_{\Omega} q_{\mu} C_{i j k l, \mu} u_{k, l} u_{i, j} d x+ \\
& \quad+\int_{\Omega}\left(\alpha_{i j} \theta\right)_{j} q_{\mu} u_{i, \mu} d x+\int_{\Omega}\left(\beta_{i j} P\right)_{, j} q_{\mu} u_{i, \mu} d x .
\end{aligned}
$$

Поскольку на $\Gamma_{1}$ выполнено равенство $u_{i}=0$, получаем

$$
\nu_{j} u_{i, \mu}=\nu_{\mu} u_{i, j}
$$

Теперь оценим первый и третий интеграл правой части (4.6): 


$$
\begin{aligned}
\int_{\Gamma} \nu_{j} C_{i j k l} u_{k, l} q_{\mu} u_{i, \mu} d \Gamma-\frac{1}{2} \int_{\Gamma}\left(q_{\mu} \cdot \nu_{\mu}\right) C_{i j k l} u_{k, l} u_{i, j} d \Gamma=\int_{\Gamma_{0}} q_{\mu} u_{i, \mu} C_{i j k l} u_{k, l} \nu_{j} d \Gamma+ \\
\quad+\frac{1}{2} \int_{\Gamma_{1}}\left(q_{\mu} \cdot \nu_{\mu}\right) C_{i j k l} u_{k, l} u_{i, j} d \Gamma-\frac{1}{2} \int_{\Gamma_{0}}\left(q_{\mu} \cdot \nu_{\mu}\right) C_{i j k l} u_{k, l} u_{i, j} d \Gamma .
\end{aligned}
$$

Подстановка (4.8) в (4.6) дает

$$
\begin{aligned}
& \int_{\Omega} \rho \ddot{u}_{i} q_{\mu} u_{i, \mu} d x=\int_{\Gamma_{0}} q_{\mu} u_{i, \mu} C_{i j k l} u_{k, l} \nu_{j} d \Gamma+\frac{1}{2} \int_{\Gamma_{1}}\left(q_{\mu} \cdot \nu_{\mu}\right) C_{i j k l} u_{k, l} u_{i, j} d \Gamma- \\
& -\frac{1}{2} \int_{\Gamma_{0}}\left(q_{\mu} \cdot \nu_{\mu}\right) C_{i j k l} u_{k, l} u_{i, j} d \Gamma-\int_{\Omega} C_{i j k l} u_{k, l} u_{i, j} d x+\frac{n}{2} \int_{\Omega} C_{i j k l} u_{k, l} u_{i, j} d x+ \\
& \quad+\frac{1}{2} \int_{\Omega} q_{\mu} C_{i j k l, \mu} u_{k, l} u_{i, j} d x+\int_{\Omega}\left(\alpha_{i j} \theta\right)_{j} q_{\mu} u_{i, \mu} d x+\int_{\Omega}\left(\beta_{i j} P\right)_{, j} q_{\mu} u_{i, \mu} d x .
\end{aligned}
$$

Подстановка (4.3) и (4.9) в (4.2) дает (4.1).

Введем следующие функционалы:

$$
\mathcal{G}(t)=\int_{\Omega} \rho \dot{u}_{i} u_{i} d x, \quad \mathcal{H}(t)=\mathcal{F}(t)+(n-1) \mathcal{G}(t) .
$$

Непосредственное вычисление дает

$$
\frac{d}{d t} \mathcal{G}(t)=\int_{\Omega} \rho\left|\dot{u}_{i}\right|^{2} d x+\int_{\Gamma_{0}} C_{i j k l} u_{k, l} \nu_{, j} u_{i} d \Gamma-\int_{\Omega} C_{i j k l} u_{k, l} u_{i, j} d x-\int_{\Omega}\left(\alpha_{i j} \theta+\beta_{i j} P\right) u_{i, j} d x .
$$

Лемма 4.2. Для любого начального условия $\left(u^{0}, u^{1}, \theta^{0}, P^{0}\right) \in \mathcal{H}$, решение $(u, v, \theta, P)$ задачи (2.6)-(2.9) удовлетворяет неравенству

$$
\begin{aligned}
\frac{d}{d t} \mathcal{H}(t) \leqslant & -\gamma_{0} \int_{\Omega}\left|\dot{u}_{i}\right|^{2} d x-\left(\frac{\gamma_{1}}{2}-\epsilon \lambda_{1}\right) \int_{\Omega}\left|u_{i, j}\right|^{2} d x+\tau \int_{\Omega}\left(\left|\theta_{, j}\right|^{2}+\left|P_{, j}\right|^{2}\right) d x+ \\
& +\left(\frac{(n-1)^{2}}{2 \epsilon g^{2}(0)}+\rho_{1} a_{1}+\frac{R_{0}^{2}}{2 \epsilon g^{2}(0)}\right) \int_{\Gamma_{0}}\left|\dot{u}_{i}\right|^{2} d \Gamma+\left(\frac{(n-1)^{2}}{2 \epsilon}+\frac{R_{0}^{2}}{2 \epsilon}\right) \int_{\Gamma_{0}}\left|r * \dot{u}_{i}\right|^{2} d \Gamma- \\
& \quad-\left(a_{0}-\frac{\epsilon}{\alpha}\right) \int_{\Gamma_{0}} C_{i j k l} u_{k, l} u_{i, j} d \Gamma,
\end{aligned}
$$

где $\gamma_{0}$ и $\gamma_{1}$ определены в (2.14c) и (2.11a), $\epsilon$ - положительная константа, которая будет определена позже, $\tau=\tau_{1}+\tau_{2}$,

$$
\tau_{1}=\max \left\{\frac{2(n-1)^{2} \omega_{1}^{2} C_{p}}{\gamma_{1}}, \frac{2(n-1)^{2} \omega_{2}^{2} C_{p}}{\gamma_{1}}\right\}, \quad \tau_{2}=\max \left\{\frac{8 R_{0}^{2}\left(\omega_{3}^{2} C_{p}+\omega_{1}\right)}{\gamma_{1}}, \frac{8 R_{0}^{2}\left(\omega_{4}^{2} C_{p}+\omega_{2}\right)}{\gamma_{1}}\right\} .
$$

Доказательство. Дифференцируя функцию $\mathcal{H}(t)$ по времени и используя (4.10) и (4.1), получаем

$$
\begin{aligned}
\frac{d}{d t} \mathcal{H}(t) & =\int_{\Gamma_{0}} \rho\left(q_{\mu} \cdot \nu_{\mu}\right)\left|\dot{u}_{i}\right|^{2} d \Gamma-\int_{\Omega}\left(\rho+\rho_{, \mu} q_{\mu}\right)\left|\dot{u}_{i}\right|^{2} d x-\int_{\Omega}\left(C_{i j k l}-q_{\mu} C_{i j k l, \mu}\right) u_{k, l} u_{i, j} d x+ \\
& +2 \int_{\Gamma_{0}} q_{\mu} u_{i, \mu} C_{i j k l} u_{k, l} \nu_{j} d \Gamma+\int_{\Gamma_{1}}\left(q_{\mu} \cdot \nu_{\mu}\right) C_{i j k l} u_{k, l} u_{i, j} d \Gamma-\int_{\Gamma_{0}}\left(q_{\mu} \cdot \nu_{\mu}\right) C_{i j k l} u_{k, l} u_{i, j} d \Gamma+
\end{aligned}
$$




$$
\begin{aligned}
+(n-1) \int_{\Gamma_{0}} C_{i j k l} u_{k, l} \nu_{, j} u_{i} d \Gamma & -(n-1) \int_{\Omega}\left(\alpha_{i j} \theta+\beta_{i j} P\right) u_{i, j} d x+ \\
& +2 \int_{\Omega}\left(\alpha_{i j} \theta\right)_{j} q_{\mu} u_{i, \mu} d x+2 \int_{\Omega}\left(\beta_{i j} P\right)_{, j} q_{\mu} u_{i, \mu} d x .
\end{aligned}
$$

В силу (2.3) и (2.14b) первое слагаемое правой части (4.13) допускает следующую оценку:

$$
\int_{\Gamma_{0}} \rho\left(q_{\mu} \cdot \nu_{\mu}\right)\left|\dot{u}_{i}\right|^{2} d \Gamma \leqslant \rho_{1} a_{1} \int_{\Gamma_{0}}\left|\dot{u}_{i}\right|^{2} d \Gamma .
$$

В силу (2.11a) и (2.14c) второе и третье слагаемые правой части (4.13) допускают следующую оценку:

$$
-\int_{\Omega}\left(\rho+\rho_{, \mu} q_{\mu}\right)\left|\dot{u}_{i}\right|^{2} d x-\int_{\Omega}\left(C_{i j k l}-q_{\mu} C_{i j k l, \mu}\right) u_{k, l} u_{i, j} d x \leqslant-\gamma_{0} \int_{\Omega}\left|\dot{u}_{i}\right|^{2} d x-\gamma_{1} \int_{\Omega}\left|u_{i, j}\right|^{2} d x .
$$

В силу (2.11b), (2.17) и неравенства Юнга четвертое слагаемое правой части (4.13) допускает оценку

$$
\begin{aligned}
\int_{\Gamma_{0}} q_{\mu} u_{i, \mu} C_{i j k l} u_{k, l} \nu_{j} d \Gamma \leqslant \epsilon \int_{\Gamma_{0}}\left|u_{i, j}\right|^{2} d \Gamma+\frac{R_{0}^{2}}{2 \epsilon g^{2}(0)} \int_{\Gamma_{0}}\left|\dot{u}_{i}\right|^{2} d \Gamma+\frac{R_{0}^{2}}{2 \epsilon} \int_{\Gamma_{0}}\left|r * \dot{u}_{i}\right|^{2} d \Gamma \leqslant \\
\quad \leqslant \frac{\epsilon}{\alpha} \int_{\Gamma_{0}} C_{i j k l} u_{k, l} u_{i, j} d \Gamma+\frac{R_{0}^{2}}{2 \epsilon g^{2}(0)} \int_{\Gamma_{0}}\left|\dot{u}_{i}\right|^{2} d \Gamma+\frac{R_{0}^{2}}{2 \epsilon} \int_{\Gamma_{0}}\left|r * \dot{u}_{i}\right|^{2} d \Gamma .
\end{aligned}
$$

Используя (2.1) и (2.11b), получаем

$$
\int_{\Gamma_{1}}\left(q_{\mu} \cdot \nu_{\mu}\right) C_{i j k l} u_{k, l} u_{i, j} d \Gamma \leqslant 0 .
$$

Следовательно, пятое и шестое слагаемые правой части (4.13) допускают оценку

$$
\begin{aligned}
\int_{\Gamma_{1}}\left(q_{\mu} \cdot \nu_{\mu}\right) C_{i j k l} u_{k, l} u_{i, j} d \Gamma-\int_{\Gamma_{0}}\left(q_{\mu} \cdot \nu_{\mu}\right) C_{i j k l} u_{k, l} u_{i, j} d \Gamma \leqslant \\
\leqslant-\int_{\Gamma_{0}}\left(q_{\mu} \cdot \nu_{\mu}\right) C_{i j k l} u_{k, l} u_{i, j} d \Gamma \leqslant(\text { в силу }(2.3)) \leqslant-a_{0} \int_{\Gamma_{0}} C_{i j k l} u_{k, l} u_{i, j} d \Gamma .
\end{aligned}
$$

В силу (2.17) и неравенства Юнга седьмое слагаемое правой части (4.13) допускает оценку

$$
\begin{gathered}
(n-1) \int_{\Gamma_{0}} C_{i j k l} u_{k, l} \nu_{, j} u_{i} d \Gamma \leqslant \epsilon \int_{\Gamma_{0}}\left|u_{i}\right|^{2} d \Gamma+\frac{(n-1)^{2}}{2 \epsilon g^{2}(0)} \int_{\Gamma_{0}}\left|\dot{u}_{i}\right|^{2} d \Gamma+\frac{(n-1)^{2}}{2 \epsilon} \int_{\Gamma_{0}}\left|r * \dot{u}_{i}\right|^{2} d \Gamma \leqslant \\
\leqslant(\text { в силу }(3.2)) \leqslant \epsilon \lambda_{1} \int_{\Omega}\left|u_{i, j}\right|^{2} d x+\frac{(n-1)^{2}}{2 \epsilon g^{2}(0)} \int_{\Gamma_{0}}\left|\dot{u}_{i}\right|^{2} d \Gamma+\frac{(n-1)^{2}}{2 \epsilon} \int_{\Gamma_{0}}\left|r * \dot{u}_{i}\right|^{2} d \Gamma .
\end{gathered}
$$

В силу неравенства Юнга и (2.13) три последних слагаемых правой части (4.13) допускают оценку

$$
\begin{gathered}
-(n-1) \int_{\Omega}\left(\alpha_{i j} \theta+\beta_{i j} P\right) u_{i, j} d x \leqslant \tau_{1} \int_{\Omega}\left(\theta_{, j}^{2}+P_{, j}^{2}\right) d x+\frac{\gamma_{1}}{4} \int_{\Omega}\left|u_{i, j}\right|^{2} d x \\
2 \int_{\Omega} q_{\mu} u_{i, \mu}\left(\alpha_{i j} \theta\right)_{j} d x+2 \int_{\Omega} q_{\mu} u_{i, \mu}\left(\beta_{i j} P\right)_{j} d x \leqslant \tau_{2} \int_{\Omega}\left(\theta_{, j}^{2}+P_{, j}^{2}\right) d x+\frac{\gamma_{1}}{4} \int_{\Omega}\left|u_{i, j}\right|^{2} d x,
\end{gathered}
$$

где $\tau_{1}$ и $\tau_{2}$ определены в (4.12). Подстановка (4.14)-(4.20) в (4.18) дает (4.11).

Теперь есть все необходимое, чтобы доказать основной результат этого раздела. 
Доказательство теоремы 2.1. Зададим функцию Ляпунова

$$
\mathcal{L}(t)=\delta E(t)+\mathcal{H}(t),
$$

где $\delta$ - положительная константа, определенная ниже. Используя (2.4), (2.14b) и (2.11b), получаем

$$
|\mathcal{H}(t)| \leqslant \int_{\Omega}\left|2 \rho\left(q_{\mu} u_{i, \mu}\right) \dot{u}_{i}\right| d x+(n-1) \int_{\Omega}\left|\rho \dot{u}_{i} u_{i}\right| d x \leqslant \varrho_{0} \int_{\Omega}\left(\rho\left|\dot{u}_{i}\right|^{2}+C_{i j k l} u_{i, j} u_{k, l}\right) d x \leqslant \varrho_{0} E(t),
$$

где $\varrho_{0}$ определено в (2.27). Таким образом, легко показать, что

$$
\left(\delta-\varrho_{0}\right) E(t) \leqslant \mathcal{L}(t) \leqslant\left(\delta+\varrho_{0}\right) E(t) .
$$

Из (3.7) и (4.11) следует

$$
\begin{aligned}
& \frac{d}{d t} \mathcal{L}(t) \leqslant-\gamma_{0} \int_{\Omega}\left|\dot{u}_{i}\right|^{2} d x-\left(\frac{\gamma_{1}}{2}-\epsilon \lambda_{1}\right) \int_{\Omega}\left|u_{i, j}\right|^{2} d x-\left(\frac{\delta}{g(0)}-\frac{(n-1)^{2}}{2 \epsilon g^{2}(0)}-\rho_{1} a_{1}-\frac{R_{0}^{2}}{2 \epsilon}\right) \int_{\Gamma_{0}}\left|\dot{u}_{i}\right|^{2} d \Gamma- \\
&-\left(\frac{(n-1)^{2}}{2 \epsilon g^{2}(0)}-\frac{R_{0}^{2}}{2 \epsilon g^{2}(0)}\right) \int_{\Gamma_{0}}\left|r * \dot{u}_{i}\right|^{2} d \Gamma-\delta \int_{\Gamma_{0}}\left(r * \dot{u}_{i}\right) \dot{u}_{i} d \Gamma-(\delta \varsigma-\tau) \int_{\Omega}\left|\theta_{, j}\right|^{2} d x- \\
&-(\delta \kappa-\tau) \int_{\Omega}\left|P_{, j}\right|^{2} d x-\left(a_{0}-\frac{\epsilon}{\alpha}\right) \int_{\Gamma_{0}} C_{i j k l} u_{k, l} u_{i, j} d \Gamma .
\end{aligned}
$$

Интегрируя это неравенство на $\left(t_{0}, t_{1}\right)$ и используя $(2.21)$, получаем

$$
\begin{aligned}
& \int_{t_{0}}^{t_{1}} \frac{d}{d t} \mathcal{L}(t) d t \leqslant-\gamma_{0} \int_{t_{0}}^{t_{1}} \int_{\Omega}\left|\dot{u}_{i}\right|^{2} d x d t- \\
& -\left(\frac{\gamma_{1}}{2}-\epsilon \lambda_{1}\right) \int_{t_{0}}^{t_{1}} \int_{\Omega}\left|u_{i, j}\right|^{2} d x d t-\left(\frac{\delta}{g(0)}-\frac{(n-1)^{2}}{4 \epsilon g^{2}(0)}-\rho_{1} a_{1}-\frac{R_{0}^{2}}{2 \epsilon}\right) \int_{t_{0}}^{t_{1}} \int_{\Gamma_{0}}\left|\dot{u}_{i}\right|^{2} d \Gamma d t- \\
& -\left(\frac{\delta}{\mathfrak{B}_{r} \mathfrak{B}_{0}}+\frac{(n-1)^{2}}{4 \epsilon g^{2}(0)}-\frac{R_{0}^{2}}{2 \epsilon g^{2}(0)}\right) \int_{\Gamma_{0}} \int_{t_{0}}^{t_{1}}\left|r * \dot{u}_{i}\right|^{2} d t d \Gamma-(\delta \varsigma-\tau) \int_{t_{0}}^{t_{1}} \int_{\Omega}\left|\theta_{, j}\right|^{2} d x d t- \\
& \quad-(\delta \kappa-\tau) \int_{t_{0}}^{t_{1}} \int_{\Omega}\left|P_{, j}\right|^{2} d x d t-\left(a_{0}-\frac{\epsilon}{\alpha}\right) \int_{t_{0}}^{t_{1}} \int_{\Gamma_{0}} C_{i j k l} u_{k, l} u_{i, j} d \Gamma d t
\end{aligned}
$$

где $\mathfrak{B}_{0}$ и $\mathfrak{B}_{r}$ определены в лемме 2.1. Выберем $\delta$ и $\epsilon$ (аналогично $(2.27)$ ) так, чтобы выражения

$$
\begin{gathered}
\frac{\gamma_{1}}{2}-\epsilon \lambda_{1}, \quad \frac{\delta}{g(0)}-\frac{(n-1)^{2}}{4 \epsilon g^{2}(0)}-\rho_{1} a_{1}-\frac{R_{0}^{2}}{2 \epsilon}, \\
\frac{\delta}{\mathfrak{B}_{r} \mathfrak{B}_{0}}+\frac{(n-1)^{2}}{4 \epsilon g^{2}(0)}-\frac{R_{0}^{2}}{2 \epsilon g^{2}(0)}, \quad \delta \varsigma-\tau, \quad \delta \kappa-\tau, \quad a_{0}-\frac{\epsilon}{\alpha}
\end{gathered}
$$

были положительны. Следовательно, вышеуказанные интегралы могут быть опущены, и (4.23) примет вид

$$
\begin{aligned}
\mathcal{L}\left(t_{1}\right)-\mathcal{L}\left(t_{0}\right) \leqslant-\gamma_{0} & \int_{t_{0}}^{t_{1}} \int_{\Omega}\left|\dot{u}_{i}\right|^{2} d x d t-\left(\frac{\gamma_{1}}{2}-\epsilon \lambda_{1}\right) \int_{t_{0}}^{t_{1}} \int_{\Omega}\left|u_{i, j}\right|^{2} d x d t- \\
& \quad-(\delta \varsigma-\tau) \int_{t_{0}}^{t_{1}} \int_{\Omega}\left|\theta_{, j}\right|^{2} d x d t-(\delta \kappa-\tau) \int_{t_{0}}^{t_{1}} \int_{\Omega}\left|P_{, j}\right|^{2} d x d t \leqslant-\Lambda_{1} \int_{t_{0}}^{t_{1}} E(s) d s,
\end{aligned}
$$


$\left(\Lambda_{1}\right.$ определено в $\left.(2.27)\right)$. Поскольку указанное неравенство справедливо для всех $t_{0} \leqslant t_{1}$, то $\mathcal{L}(t)$ - невозрастающая функция. Выбирая $t_{0}=t$ и $t_{1}=t+1$ в $(4.25)$, получаем

$$
\mathcal{L}(t+1)-\mathcal{L}(t) \leqslant-\Lambda_{1} \int_{t}^{t+1} E(s) d s \leqslant(\text { в силу }(4.22)) \leqslant-\frac{\Lambda_{1}}{\delta+\varrho_{0}} \int_{t}^{t+1} \mathcal{L}(s) d s .
$$

Поскольку $\mathcal{L}(s)$ - невозрастающая функция при $t \leqslant s \leqslant t+1$, то верно неравенство

$$
\mathcal{L}(t+1)-\mathcal{L}(t) \leqslant-\frac{\Lambda_{1}}{\delta+\varrho_{0}} \mathcal{L}(t+1) .
$$

Итак, получаем

$$
\max _{t \leqslant s \leqslant t+1}\{\mathcal{L}(t)\}=\mathcal{L}(t)=\mathcal{L}(t)-\mathcal{L}(t+1)+\mathcal{L}(t+1)
$$

Из (4.27) следует

$$
\mathcal{L}(t+1) \leqslant \frac{\delta+\varrho_{0}}{\Lambda_{1}}(\mathcal{L}(t)-\mathcal{L}(t+1))
$$

Подстановка этого неравенства в (4.28) дает

$$
\max _{t \leqslant s \leqslant t+1}\{\mathcal{L}(t)\} \leqslant\left(1+\frac{\delta+\varrho_{0}}{\Lambda_{1}}\right)(\mathcal{L}(t)-\mathcal{L}(t+1)) .
$$

Это означает, что $\mathcal{L}(t)$ удовлетворяет неравенству Накао $(2.23)\left(\right.$ для $\left.\varpi=\left(\Lambda_{1}+\delta+\varrho_{0}\right) / \Lambda_{1}\right)$. Получаем

$$
\mathcal{L}(t) \leqslant \mathcal{L}(0) \exp \left(\frac{-\Lambda_{1}}{\Lambda_{1}+\delta+\varrho_{0}} t\right)
$$

Используя (4.22), получаем

$$
\mathcal{L}(t) \leqslant\left(\delta+\varrho_{0}\right) E(0) \exp \left(\frac{-\Lambda_{1}}{\Lambda_{1}+\delta+\varrho_{0}} t\right) .
$$

Повторное использование (4.22) влечет (2.26).

5. Управляемость. В этом разделе с помощью метода единственности Гильберта (см. [18]) и принципа Расселла «управляемость через стабилизируемость» (см. [31]) доказана частичная точная управляемость задачи (2.6)-(2.7) со следующими однородными начальными условиями:

$$
u(x, 0)=0, \quad \dot{u}(x, 0)=0, \quad \theta(x, 0)=0, \quad P(x, 0)=0, \quad x \in \Omega,
$$

где $\phi=\left(\phi_{1}, \ldots, \phi_{n}\right)$ в $(2.7 \mathrm{~b})$ - неизвестная функция управления (которая будет определена позже), действующая на границе $\Gamma_{0}$. При заданных $T>0, Q=\Omega \times(0, T), \Sigma=\Gamma \times(0, T)$, $\Sigma_{0}=\Gamma_{0} \times(0, T)$ и $\Sigma_{1}=\Gamma_{1} \times(0, T)$ частичная точная управляемость определяется следующим способом (см. $[6,18,22,26])$.

Определение 5.1. Задача (2.6)-(2.7) с начальными условиями (5.1) называется частично точно управляемой, если существует такое $T>0$, что для каждого состояния $\left(u^{0}, u^{1}\right) \in W$ найдется управление $\phi \in\left(L^{2}\left(\Sigma_{0}\right)\right)^{n}$, для которого соответствующее решение удовлетворяет условиям

$$
u(x, T)=u^{0}(x), \quad \dot{u}(x, T)=u^{1}(x), \quad x \in \Omega,
$$

независимо от значений температуры и химического потенциала.

Как указано в [18, с. 34-35], это эквивалентно приведению каждого начального состояния $\left(u_{0}, u_{1}\right)$ в состояние $(u(T), \dot{u}(T))=(0,0)$ независимо от значений температуры и химического потенциала. 
Теперь сформулируем следующую задачу Ламе:

$$
\begin{cases}\rho \ddot{z}_{i}=\left(C_{i j k l} z_{k, l}\right)_{, j} & \text { в } Q, \\ z_{i}=0 & \text { на } \Sigma_{1}, \\ z_{i}=g * C_{i j k l} z_{k, l} \nu_{j} & \text { на } \Sigma_{0}, \\ z_{i}(T)=z_{i}^{0}, \dot{z}_{i}(T)=z_{i}^{1} & \text { в } \Omega,\end{cases}
$$

где $\left(z^{0}, z^{1}\right) \in W$. Из классической теории полугрупп (см. [29] и [30, теорема 4.4]) следует, что задача (5.3) имеет единственное решение $(z(t), \dot{z}(t)) \in C(0, T ; W)$. Поскольку задача (5.3) является частным случаем задачи (2.6)-(2.8), то, используя ту же процедуру, можем привести соотношение (2.17) к виду

$$
C_{i j k l} z_{k, l} \nu_{j}=\frac{1}{g(0)} \dot{z}_{i}+r * \dot{z}_{i}
$$

и, следовательно, энергия (5.3), имеющая вид

$$
E_{L}(z, t)=\frac{1}{2} \int_{\Omega}\left(\rho \dot{z}_{i}^{2}+C_{i j k l} z_{k, l} z_{i, j}\right) d x,
$$

становится возрастающей функцией, удовлетворяющей уравнению

$$
E_{L}(z, T)-E_{L}(z, 0)=\int_{\Sigma_{0}}\left(\frac{1}{g(0)} \dot{z}_{i}^{2}+\left(r * \dot{z}_{i}\right) \dot{z}_{i}\right) d \Sigma>0 .
$$

Кроме того, существует такая положительная константа $\omega$, что

$$
E_{L}(z, t) \leqslant E_{L}(z, T) e^{1-\omega(T-t)} \quad \text { для всех } t \in[0, T] .
$$

Используя решение задачи Ламе (5.3), рассмотрим следующую термодиффузионную задачу:

$$
\begin{cases}c \dot{\xi}+d \dot{\chi}=-\alpha_{i j} \dot{z}_{i, j}+\left(k_{i j} \xi_{, j}\right)_{, i} & \text { в } Q, \\ d \dot{\xi}+r \dot{\chi}=-\beta_{i j} \dot{z}_{i, j}+\left(h_{i j} \chi_{, j}\right)_{, i} & \text { в } Q, \\ \xi=\chi=0 & \text { на } \Sigma, \\ \xi(0)=\chi(0)=0 & \text { в } \Omega .\end{cases}
$$

Поскольку $\dot{z}_{i, j} \in\left(L^{2}\left(0, T ; H^{-1}(\Omega)\right)\right)^{n}$, из классической теории полугрупп следует, что задача $(5.8)$ имеет единственное решение

$$
(\xi, \chi) \in\left(C\left(0, T ; L^{2}(\Omega)\right) \cap L^{2}\left(0, T ; H_{0}^{1}(\Omega)\right)\right)^{2} .
$$

В дальнейшем нам понадобятся следующие леммы.

Лемма 5.1. Пусть выполнено условие (2.15) и ( $(, \chi)$ является решением задачи (5.8). Тогда

$$
\int_{0}^{T}\left\|\left(\alpha_{i j} \xi+\beta_{i j} \chi\right)_{, j}\right\|_{L^{2}(\Omega)}^{2} d t \leqslant \frac{\eta^{2} \gamma^{2} \sigma^{2} e}{\omega} E_{L}(z, T),
$$

где

$$
\eta^{2}=2 \frac{\left(\omega_{3} C_{p}+\omega_{1}\right)^{2}}{\zeta}+2 \frac{\left(\omega_{4} C_{p}+\omega_{2}\right)^{2}}{\kappa}, \quad \gamma^{2}=\max \left\{\frac{\omega_{1}^{2}+\omega_{3}^{2} C_{p}}{\zeta}, \frac{\omega_{2}^{2}+\omega_{4}^{2} C_{p}}{\kappa}\right\},
$$

$\sigma$ определена в $(2.5), \omega_{1}, \omega_{2}, \omega_{3}, \omega_{4}$ определены в (2.13).

Доказательство этой леммы аналогично доказательству [3, лемма 5.2]. 
Теперь, используя решение $(\xi, \chi)$ задачи (5.8) и начальные условия задачи (5.3), рассмотрим следующую задачу:

$$
\begin{cases}\rho \ddot{w}_{i}=\left(C_{i j k l} w_{k, l}+\alpha_{i j} \varphi+\beta_{i j} \psi\right)_{, j}+\left(\alpha_{i j} \xi+\beta_{i j} \chi\right)_{, j} & \text { в } Q, \\ c \dot{\varphi}+d \dot{\psi}=\alpha_{i j} \dot{w}_{i, j}+\left(k_{i j} \varphi_{, j}\right)_{, i} & \text { в } Q, \\ d \dot{\varphi}+r \dot{\psi}=\beta_{i j} \dot{w}_{i, j}+\left(h_{i j} \psi_{, j}\right)_{, i} & \text { в } Q, \\ w_{i}=0 & \text { на } \Sigma_{1}, \\ w_{i}(x, t)+g * C_{i j k l} w_{k, l} \nu_{j}=0 & \text { на } \Sigma_{0}, \\ \varphi=\psi=0 & \text { на } \Sigma, \\ w_{i}(x, 0)=z_{i}(0), \dot{w}_{i}(x, 0)=\dot{z}_{i}(0), \varphi(x, 0)=0, \psi(x, 0)=0, & x \in \Omega .\end{cases}
$$

Поскольку $(\xi, \chi) \in\left(L^{2}\left(0, T ; H_{0}^{1}(\Omega)\right)\right)^{2}$, из $(5.9)$ следует, что

$$
\left(0,-\left(\alpha_{i j} \xi+\beta_{i j} \chi\right), j, 0,0\right) \in L^{1}(0, T ; \mathcal{H}) .
$$

Поскольку $(w(0), \dot{w}(0), \varphi(0), \psi(0)) \in \mathcal{H}$, из классической теории полугрупп следует, что неоднородная задача (5.11) имеет единственное решение $(w, \dot{w}, \varphi, \psi) \in C(0, T ; \mathcal{H})$, которое может быть записано в виде

$$
\left(w_{i}, \dot{w}_{i}, \varphi, \psi\right)=S(t)\left(w_{i}(0), \dot{w}_{i}(0), \varphi(0), \psi(0)\right)+\int_{0}^{t} S(t-\tau)\left(0,-\left(\alpha_{i j} \xi+\beta_{i j} \chi\right)_{, j}, 0,0\right) d \tau
$$

где $S(t)$ обозначает сильно непрерывную полугруппу сжатий, порожденную оператором $\mathcal{A}$, заданным в (3.3). В силу равенства $E(t)=2\|U(t)\|^{2}=2\|S(t) U(0)\|^{2}$ из теоремы 2.1 получаем, что

$$
\|S(t)\| \leqslant \Lambda^{1 / 2} e^{-\omega t / 2} \leqslant \Lambda^{1 / 2} e^{(1-\omega t) / 2} \quad \text { для всех } t \geqslant 0 .
$$

Таким образом, объединяя (5.12) и (5.13), заключаем, что энергия $\mathcal{E}(t)$ в задаче $(5.11)$ удовлетворяет неравенству

$$
\begin{gathered}
\mathcal{E}(t) \leqslant 2\|S(t)\|^{2} \mathcal{E}(0)+2\left(\int_{0}^{t}\|S(t-\tau)\|\left\|\left(\alpha_{i j} \xi+\beta_{i j} \chi\right), j\right\|_{L^{2}(\Omega)} d \tau\right)^{2} \leqslant \\
\leqslant 2 \Lambda e^{1-\omega t} \mathcal{E}(0)+2 \Lambda \int_{0}^{t} e^{1-\omega(t-\tau)} d \tau \int_{0}^{t}\left\|\left(\alpha_{i j} \xi+\beta_{i j} \chi\right)_{, j}\right\|_{L^{2}(\Omega)}^{2} d \tau \leqslant \\
\leqslant(\text { в силу }(5.9)) \leqslant 2 \Lambda e^{1-\omega t} \mathcal{E}(0)+4 \frac{\Lambda \eta^{2} \gamma^{2} \sigma^{2} e^{2}}{\omega^{2}} E_{L}(z, T),
\end{gathered}
$$

где $\mathcal{E}(t)$ также определена в $(2.25)$.

Лемма 5.2. Пусть ( $z, \dot{z})$ - решение задачи Ламе (5.3) u $(w, \dot{w}, \varphi, \psi)$ - решение задачи (5.11). Тогда существует такая положительная константа $c_{1}$, что

$$
\mathcal{E}(T)+\int_{0}^{T}\left(\varsigma\left\|\varphi_{, i}\right\|_{L^{2}(\Omega)}^{2}+\kappa\left\|\psi_{, i}\right\|_{L^{2}(\Omega)}^{2}\right) d t+\frac{1}{g(0)} \int_{\Sigma_{0}} \dot{w}_{i}^{2} d \Sigma+\int_{\Sigma_{0}}\left(r * \dot{w}_{i}\right) \dot{w}_{i} d \Sigma \leqslant c_{1} E_{L}(z, T),
$$

əde

$$
c_{1}=1+2 \sigma^{2} \Lambda \frac{e}{\omega}+\frac{\eta^{2} \gamma^{2} \sigma^{2} e}{2 \omega}+\frac{4 T \Lambda \eta^{2} \gamma^{2} \sigma^{4} e^{2}}{\omega^{2}}
$$


Доказательство. Умножая (5.11a) на $\dot{w}_{i},(5.11 \mathrm{~b})$ на $\varphi,(5.11 \mathrm{c})$ на $\psi$, интегрируя, суммируя и используя (2.12), получаем

$$
\begin{aligned}
\mathcal{E}(T)+\int_{0}^{T}\left(\varsigma\left\|\varphi_{, i}\right\|_{L^{2}(\Omega)}^{2}+\kappa\left\|\psi_{, i}\right\|_{L^{2}(\Omega)}^{2}\right) d t+\frac{1}{g(0)} \int_{\Sigma_{0}} \dot{w}_{i}^{2} d \Sigma+\int_{\Sigma_{0}}\left(r * \dot{w}_{i}\right) \dot{w}_{i} d \Sigma \leqslant & \\
& \leqslant \mathcal{E}(0)-\int_{0}^{T} \int_{\Omega}\left(\alpha_{i j} \xi+\beta_{i j} \chi\right) \dot{w}_{i, j} d x d t .
\end{aligned}
$$

В силу неравенства Юнга последнее слагаемое правой части (5.16) допускают оценку

$$
\begin{gathered}
-\int_{0}^{T} \int_{\Omega}\left(\alpha_{i j} \xi+\beta_{i j} \chi\right) \dot{w}_{i, j} d x d t \leqslant \int_{0}^{T}\left\|\left(\alpha_{i j} \xi+\beta_{i j} \chi\right)_{, j}\right\|_{L^{2}(\Omega)} \|\left.\dot{w}_{i, j}\right|_{-1} d t \leqslant(\text { в силу }(2.5)) \leqslant \\
\leqslant \frac{1}{2} \int_{0}^{T}\left\|\left.\left(\alpha_{i j} \xi+\beta_{i j} \chi\right)_{, j}\right|_{L^{2}} ^{2} d t+\frac{\sigma^{2}}{2} \int_{0}^{T}\right\| \dot{w} \| d t \leqslant(\text { в силу }(5.9)) \leqslant \\
\leqslant \frac{\eta^{2} \gamma^{2} \sigma^{2} e}{2 \omega} E_{L}(z, T)+\sigma^{2} \int_{0}^{T} \mathcal{E}(t) d t \leqslant(\text { в силу }(5.14)) \leqslant \\
\leqslant\left(\frac{\eta^{2} \gamma^{2} \sigma^{2} e}{2 \omega}+4 T \sigma^{2} \frac{\Lambda \eta^{2} \gamma^{2} \sigma^{2} e^{2}}{\omega^{2}}\right) E_{L}(z, T)+2 \sigma^{2} \Lambda \frac{e}{\omega} \mathcal{E}(0) .
\end{gathered}
$$

Подставляя это неравенство в (5.16) и используя неравенство $\mathcal{E}(0)=E_{L}(z, 0)<E_{L}(z, T)$, завершаем доказательство.

Теперь докажем основной результат этого раздела.

Доказательство теоремы 2.2. Положим

$$
u=w-z, \quad \theta=\varphi+\xi, \quad P=\psi+\chi,
$$

где $(w, \varphi, \psi)$ - решение задачи (5.11), $z$-решение задачи (5.3), а $(\xi, \chi)$-решение задачи (5.8). Тогда $(u, \theta, P)$ удовлетворяет следующей задаче:

$$
\begin{cases}\rho \ddot{u}_{i}=\left(C_{i j k l} u_{k, l}+\alpha_{i j} \theta+\beta_{i j} P\right)_{, j} & \text { в } Q, \\ c \dot{\theta}+d \dot{P}=\alpha_{i j} \dot{u}_{i, j}+\left(k_{i j} \theta_{, j}\right)_{, i} & \text { в } Q, \\ d \dot{\theta}+r \dot{P}=\beta_{i j} \dot{u}_{i, j}+\left(h_{i j} P_{, j}\right)_{, i} & \text { в } Q, \\ u_{i}=0 & \text { на } \Sigma_{1}, \\ u_{i}=\phi_{i} & \text { на } \Sigma_{0}, \\ \theta=P=0 & \text { на } \Sigma, \\ u_{i}(0)=\dot{u}_{i}(0)=0, \quad \theta(0)=0, \quad P(0)=0 & \text { в } \Omega, \\ u_{i}(T)=w_{i}(T)-z_{i}^{0}, \quad \dot{u}_{i}(T)=\dot{w}_{i}(T)-z_{i}^{1} & \text { в } \Omega,\end{cases}
$$

где $\phi_{i}$ задано в (2.31). Теперь определим линейный оператор

$$
\mathcal{N}: W \rightarrow W, \quad \mathcal{N}\left(z^{0}, z^{1}\right)=(w(T), \dot{w}(T)),
$$

где $\left(z^{0}, z^{1}\right) \in W$ определены в (5.3d). Кроме того, имеем

$$
\begin{aligned}
\left\|\mathcal{N}\left(z^{0}, z^{1}\right)\right\|_{W}^{2}=\| & (w(T), \dot{w}(T)) \|_{W}^{2} \leqslant \mathcal{E}(T) \leqslant \\
& \leqslant(\text { в силу }(5.14)) \leqslant 2 \Lambda e^{1-\omega T} \mathcal{E}(0)+\frac{4 \Lambda \eta^{2} \gamma^{2} \sigma^{2} e^{2}}{\omega^{2}} E_{L}(z, T)=
\end{aligned}
$$




$$
\begin{gathered}
=\left(\text { в силу соотношения } \mathcal{E}(0)=E_{L}(z, 0)<E_{L}(z, T)\right) \leqslant \\
\leqslant\left(2 \Lambda e^{(1-\omega T)}+\frac{4 \Lambda \eta^{2} \gamma^{2} \sigma^{2} e^{2}}{\omega^{2}}\right) E_{L}(z, T)=\left(2 \Lambda e^{(1-\omega T)}+\frac{4 \Lambda \eta^{2} \gamma^{2} \sigma^{2} e^{2}}{\omega^{2}}\right)\left\|\left(z^{0}, z^{1}\right)\right\|_{W}^{2},
\end{gathered}
$$

откуда следует

$$
\|\mathcal{N}\|^{2} \leqslant 2 \Lambda e^{(1-\omega T)}+\frac{4 \Lambda \eta^{2} \gamma^{2} \sigma^{2} e^{2}}{\omega^{2}}
$$

Пусть выполнено условие $(2.29)$, а $T \geqslant T_{0}$ и $T_{0}$ достаточно велики для того, чтобы выполнялось (2.30). Тогда $\|\mathcal{N}\|^{2}<1$, и $\mathcal{N}-I$ является изоморфизмом из $W$ в $W$. Таким образом, для каждого состояния $\left(u^{0}, u^{1}\right) \in W$, существует такое решение $\left(z^{0}, z^{1}\right) \in W$, что

$$
\begin{aligned}
\left(u^{0}, u^{1}\right)=\mathcal{N}\left(z^{0}, z^{1}\right)- & \left(z^{0}, z^{1}\right)=(\text { в силу }(5.20))= \\
=(w(T), \dot{w}(T))-\left(z^{0}, z^{1}\right) & =(\text { в силу }(5.18) \text { и }(5.3 \mathrm{~d}))=(u(T), \dot{u}(T)) .
\end{aligned}
$$

Следовательно, мы построили функцию управления $\phi_{i}$ заданную в $(2.31)$, определяющую решение задачи частичной точной управляемости (2.6)-(2.7) с начальными условиями (5.1).

Из (2.31) находим

$$
\left\|\phi_{i}\right\|_{L^{2}\left(\Sigma_{0}\right)}^{2}=\left\|g * C_{i j k l}\left(w_{k, l}+z_{k, l}\right) \nu_{j}\right\|_{L^{2}\left(\Sigma_{0}\right)}^{2} \leqslant 2\left\|g * C_{i j k l} w_{k, l} \nu_{j}\right\|_{L^{2}\left(\Sigma_{0}\right)}^{2}+2\left\|g * C_{i j k l} z_{k, l} \nu_{j}\right\|_{L^{2}\left(\Sigma_{0}\right)}^{2} .
$$

Используя неравенство Юнга, получаем

$$
\begin{aligned}
& \left\|\phi_{i}\right\|_{L^{2}\left(\Sigma_{0}\right)}^{2} \leqslant \\
& \leqslant 2\|g\|_{L^{1}\left(\Sigma_{0}\right)}^{2}\left\|C_{i j k l} w_{k, l} \nu_{j}\right\|_{L^{2}\left(\Sigma_{0}\right)}^{2}+2\|g\|_{L^{1}\left(\Sigma_{0}\right)}^{2}\left\|C_{i j k l} z_{k, l} \nu_{j}\right\|_{L^{2}\left(\Sigma_{0}\right)}^{2} \leqslant\left(\text { так как } g \in L_{\text {lос }}^{1}\left(\mathbb{R}^{+}\right)\right) \leqslant \\
& \quad \leqslant C \int_{\Sigma_{0}}\left|C_{i j k l} w_{k, l} \nu_{j}\right|^{2} d \Sigma+\left.C C_{i j k l} z_{k, l} \nu_{j}\right|^{2} d \Sigma \leqslant(\text { в силу }(2.17) \text { и }(5.4)) \leqslant \\
& \leqslant C\left(\frac{1}{(g(0))^{2}} \int_{\Sigma_{0}}\left|\dot{w}_{i}\right|^{2} d \Sigma+\int_{\Sigma_{0}}\left|r * \dot{w}_{i}\right|^{2} d \Sigma\right)+C\left(\frac{1}{(g(0))^{2}} \int_{\Sigma_{0}}\left|\dot{z}_{i}\right|^{2} d \Sigma+\int_{\Sigma_{0}}\left|r * \dot{z}_{i}\right|^{2} d \Sigma\right) .
\end{aligned}
$$

Поскольку резольвента $r(t)$ удовлетворяет (2.21) (см. лемму 2.1), выбирая $t_{0}=0$ и $t_{1}=T$, получаем

$$
\begin{aligned}
&\left\|\phi_{i}\right\|_{L^{2}\left(\Sigma_{0}\right)}^{2} \leqslant C\left(\frac{1}{(g(0))^{2}} \int_{\Sigma_{0}}\left|\dot{w}_{i}\right|^{2} d \Sigma+\mathfrak{B}_{0} \mathfrak{B}_{r} \int_{\Sigma_{0}}\left(r * \dot{w}_{i}\right) \dot{w}_{i} d \Sigma\right)+ \\
&+C\left(\frac{1}{(g(0))^{2}} \int_{\Sigma_{0}}\left|\dot{z}_{i}\right|^{2} d \Sigma+\mathfrak{B}_{0} \mathfrak{B}_{r} \int_{\Sigma_{0}}\left(r * \dot{z}_{i}\right) \dot{z}_{i} d \Sigma\right)\leqslant \text { (в силу }(5.15) \text { и }(5.6)) \leqslant \\
& \leqslant C E_{L}(z, T) \leqslant C\left\|\left(z_{i}^{0}, z_{i}^{1}\right)\right\|^{2} .
\end{aligned}
$$

Поскольку $\mathcal{N}-I$ является изоморфизмом $W$ в себя, из (2.30) и (5.22) следует, что $0<\|\mathcal{N}-I\| \leqslant 2$. Следовательно,

$$
\left\|\phi_{i}\right\|_{L^{2}\left(\Sigma_{0}\right)}^{2} \leqslant C\left\|(\mathcal{N}-I)^{-1}\left(u_{i}^{0}, u_{i}^{1}\right)\right\|^{2} \leqslant(\text { в силу }(5.24)) \leqslant C\left\|\left(u_{i}^{0}, u_{i}^{1}\right)\right\|^{2} .
$$

6. Заключение. 1. В статье доказаны свойства экспоненциального затухания и частичной точной управляемости для многомерной задачи термоупругой диффузии в неоднородном анизотропном материале. Свойство экспоненциального затухания установлено путем введения функции памяти на части границы и использования разностного неравенства Накао (см. [25]) при условии, что резольвента уравнения Вольтерра удовлетворяет неравенству (2.21).

2. Доказательство теоремы 2.1 опирается на свойство диссипативности рассматриваемой задачи, выполненное в силу предположения (2.21), а также на тот факт, что диссипативность в $u$ и 
$(\theta, P)$ достаточно сильна (при соблюдение условий связи), чтобы обеспечить экспоненциальную устойчивость. Другими словами, асимптотическое поведение рассматриваемой системы такое же, как у несвязанной системы (полученной путем приравнивания $\alpha_{i j}=\beta_{i j}=0$ для всех $i, j$ ). В самом деле, множители $q \nabla u_{i}$ (лемма 4.1) и $u_{i}$ (лемма 4.2) совпадают, точно так же, как для волнового уравнения в [14]. Итак, один из вкладов этой статьи состоит в том, что эти множители работают, если ядро $r$ удовлетворяет свойству (2.21) в лемме 2.1 .

3. С помощью неравенства (2.21) и метода единственности Гильберта в сочетании с принципом Расселла «управляемость через стабилизируемость» доказана частичная точная управляемость рассматриваемой системы при условии, что время управления достаточно велико, а связанные параметры напряжения-температуры и напряжения-диффузии удовлетворяют ограничению (2.29). Из условия (2.29) видно, что чем больше величина параметров напряжения-температуры и напряжения-диффузии, тем быстрее убывает энергия системы (скорость затухания $\omega$ заданная в (2.27) возрастает), и тем более система управляема. Кроме того, функция граничного управления найдена явно. Оба результата играют важную роль при выборе лучшего материала, реализующего более быстрое затухание и оптимальное время управления.

4. В [3] свойства экспоненциального затухания и управляемости установлены, когда нелинейная функция обратной связи удовлетворяет как минимум шести условиям, в то время как в этой статье требуется только, чтобы резольвента $r$ уравнения Вольтерра удовлетворяла условию (2.21). Более того, функции памяти предоставляют больше возможностей при измерении отклика материала. Это означает, что функция граничного управленияЁ(2.8) является более адекватной, чем другие граничные функции, для реализации более быстрого затухания и оптимального времени управления.

\section{СПИСОК ЛИТЕРАТУРЫ}

1. Adams R. Sobolev Spaces. - New York: Academic Press, 1975.

2. Aouadi M. Generalized theory of thermoelstic diffusion for anisotropic media// J. Therm. Stresses. — 2008. - 31. - P. 270-285.

3. Aouadi M., Boulehmi K. Partial exact controllability for inhomogeneous multidimensional thermoelastic diffusion problem// J. Evol. Equat. Control Theory. — 2016. — 5. — P. 201-224.

4. Aouadi M., Lazzari B., Nibbi R. Exponential decay in thermoelastic materials with voids and dissipative boundary without thermal dissipation// Z. Angew. Math. Phys. — 2012. - 63. - P. 961-973.

5. Aouadi M., Lazzari B., Nibbi R. Energy decay in thermoelastic diffusion theory with second sound and dissipative boundary// Mecanica. - 2013. - 48. - P. 2159-2171.

6. Apolaya R. F. Exact controllability for temporally wave equation// Portug. Math. — 1994. — 51. — P. $475-488$.

7. Barral P., Quintela P. A numerical method for simulation of thermal stresses during casting of aluminium slabs// Comput. Methods Appl. Mech. Eng. — 1998. — 178. — P. 69-88.

8. Bermudez A., Muñoz M. C., Quintela P. Numerical solution of a three-dimensional thermoelectric problem taking place in an aluminum electrolytic cell// Comput. Methods Appl. Mech. Eng. — 1993. — 106 . — P. 129-142.

9. Boulehmi K., Aouadi M. Decay of solutions in inhomogeneous thermoelastic diffusion bars// Appl. Anal. - 2014. - 93. - P. 281-304.

10. Dafermos C. M. On the existance and the asymptotic stability of solution to the equations of linear thermoelasticity// Arch. Rat. Mech. Anal. — 1968. - 29. - P. 241-271.

11. De Teresa L., Zuazua E. Controllability of the linear system of thermoelastic plates// Adv. Differ. Equations. - 1996. - 1. - P. 369-402.

12. Gao H., Muñoz Rivera J. E. On the exponential stability of thermoelastic problem with memory// Appl. Anal. - 2001. - 78. - P. 379-403.

13. Hansen $S$. W. Boundary control of a one-dimensional linear thermoelastic rod// SIAM J. Control Optim. - 1994. - 32. - P. 1054-1074.

14. Komornick V., Zuazua E. A direct method for boundary stabilisation of the wave equation// J. Math. Pures Appl. - 1990. - 69. - P. 33-54.

15. Lasiecka I. Mathematical Control Theory of Coupled PDEs. - Philadelphia: SIAM, 2002. 
16. Lebeau G., Zuazua E. Sur la décroissance non uniforme de l'énergie dans le système de la thermoélasticité linéaire// C. R. Acad. Sci. Paris. Ser. I. Math. — 1997. — 324. — P. 409-415.

17. Lebeau G., Zuazua E. Null controllability of a system of linear thermoelasticity// Arch. Rat. Mech. Anal. - 1998. - 141. - P. 297-329.

18. Lions J. L. Contôlabilité Exacte Perturbations et Stabilisations des Systèmes Distribués. Tome 2. Pertubations. - Paris: Masson, 1988.

19. Lions J. L., Magenes E. Nonhomogeneous Boundary Value Problem and Applications. — Springer-Verlag, 1972.

20. Liu W. J. Partial exact controllability and exponential stability in higher-dimensional linear thermoelasticity// ESAIM: Control Optim. Calc. Var. - 1998. - 3. - P. 23-48.

21. Liu W. J. Partial exact controllability and exponential stability in higher-dimensional linear thermoelasticity. Correction// ESAIM: Control Optim. Calc. Var. - 1998. - 3. - P. 323-327.

22. Liu W. J., Williams G. H. Partial exact controllability for the linear thermo-viscoelastic model// Electr. J. Differ. Equations. - 1998. - 1998. - P. 1-11.

23. Liu W. J., Zuazua E. Uniform stabilization of higher-dimensional system of thermoelasticity with a nonlinear boundary feedback// Quart. Appl. Math. - 2001. - 59. - P. 269-314.

24. Muñoz Rivera J. E., Olivera M. L. Stability in inhomogeneous and anisotropic thermoelasticity// Boll. U.M.I. - 1997. - 7. - P. 115-127.

25. Nakao M. A difference inequality and its application to nonlinear evolution equations// J. Math. Soc. Jpn. - 1978. - 30. - P. 291-315.

26. Nandakumaran A. K., George R. K. Partial exact controllability of linear thermoelastic system// Indian J. Math. - 1995. - 37. - P. 165-174.

27. Narukawa K. Boundary value control of thermoelastic systems// Hiroshima Math. J. — 1983. — 13. — P. 227-272.

28. Nohel J. A., Shea D. F. Frequency domain methods for Volterra equations// Adv. Math. — $1976 .-22$. - P. 278-304.

29. Prüss J. Evolutionary Integral Equations and Applications. - Basel: Birkhäuser, 1993.

30. Propst G., Prüss J. On wave equations with boundary dissipation of memory type// J. Integral Equations Appl. - 1996. - 8. - P. 99-123.

31. Russell D. L. Controllability and stabilizability theory for linear partial differential equations// Recent Progr. Open Questions SIAM Rew. - 1990. — 310. — P. 801-806.

32. Staffans O. J. An inequality for positive definite Volterra kernels// Proc. Am. Math. Soc. — 1976 . — 58. - P. 205-210.

33. Staffans O. J. On a nonlinear hyperbolic Volterra equation// SIAM J. Math.Anal. — 1980. — 11. — P. 793-812.

34. Zuazua E. Controllability of the linear system of thermoelasticity// J. Math. Pure. Appl. — 1995. - 74. - P. 303-346.

Aouadi M.

Университет Картаж, Тунис

E-mail: moncefaouadi00@gmail.com

Boulehmi K.

Университет Картаж, Тунис

E-mail: boulehmikaouther@gmail.com 